\title{
Spontaneous Acute Subdural Hematoma: Beware of the Aneurysm
}

\author{
Nathan Beucler ${ }^{1} \quad$ Christelle Haikal $^{2}$ \\ Nicolas Desse ${ }^{1} \quad$ Arnaud Dagain $^{1}$ \\ ${ }^{1}$ Departments of Neurosurgery, Sainte-Anne Military Teaching \\ Hospital, Toulon, France \\ 2Departments of Radiology, Sainte-Anne Military Teaching Hospital, \\ Toulon, France \\ ${ }^{3}$ Department of Internal Medicine, Hospitalist Service, UC San \\ Diego Medical Center, San Diego, California, United States
}

Aurore Sellier $^{1} \quad$ Christophe Joubert $^{1}$

\begin{abstract}
Address for correspondence Nathan Beucler, MD, Department of Neurosurgery, Sainte-Anne Military Teaching Hospital, 2 Boulevard SainteAnne 83000, Toulon, France (e-mail: nathan.beucler@neurochirurgie.fr).
\end{abstract}
Abstract
Keywords
- angiography
- cerebral aneurysm
- computed tomography
- digital subtraction angiography
- spontaneous acute
subdural hematoma

Spontaneous acute subdural hematoma should raise clinical suspicion for underlying pathology, the most common etiology being a ruptured aneurysm. Through this case report, our team developed a clinical decision-making tool to help physicians decide when it is necessary to order an acute subdural hematoma to assess for ruptured aneurysm.

\section{Introduction}

Acute subdural hematoma (ASH) is most frequently a consequence of traumatic brain injury (TBI). Although the most common presentation of a ruptured aneurysm is subarachnoid hemorrhage (SAH) during a hypertensive episode, in rare circumstances, the aneurysm may cause atraumatic ASH. When an aneurysm causes ASH, it is frequently associated with intracerebral hematoma (ICH) and/or SAH which helps clinicians differentiate it from ASH due to TBI.

\section{Case Report}

A 51-year-old male patient with chronic alcoholism presented to the emergency department of a local hospital for acute-onset confusion and generalized tonic-clonic seizure. His neurologic status rapidly deteriorated and he became comatose, with a Glasgow Coma Scale (GCS) of 4 with decerebrate posturing and right-pupil mydriasis. The emergency physician ordered a noncontrast head computed tomography (CT) to assess for acute bleeding with the suspicion that the patient had experienced severe TBI. The radiology report described a $12-\mathrm{mm}$ thick right ASH, causing a $10-\mathrm{mm}$ midline shift, associated with a $32 \mathrm{~mm} \times 12 \mathrm{~mm}$ intracerebral hematoma (ICH) of the right temporal pole. No evidence of skull fracture was reported ( - Fig. 1 ).

The patient was immediately transferred to our level-1 trauma center facility and presented to us 3 hours after his arrival to the emergency department. On admission, his pupil's examination was unchanged. A craniotomy for subdural hematoma evacuation was performed and revealed underneath a healthy pulsating brain. There was no external evidence of head trauma (wounds and cephalohematoma), and from our interpretation of the imaging, the temporal ICH did not look like a brain contusion. From the intraoperative exploration and the radiologic imaging, our team suspected that the patient had not sustained TBI under the influence of alcohol. To further investigate the cause of his ASH, we advised a computed tomography angiography (CTA) postoperatively. Imaging revealed a bilobed aneurysm as the source of his ICH which arose from the anastomosis between the right internal carotid artery (ICA) and the posterior communicating artery (PCOM). The aneurysm was successfully 

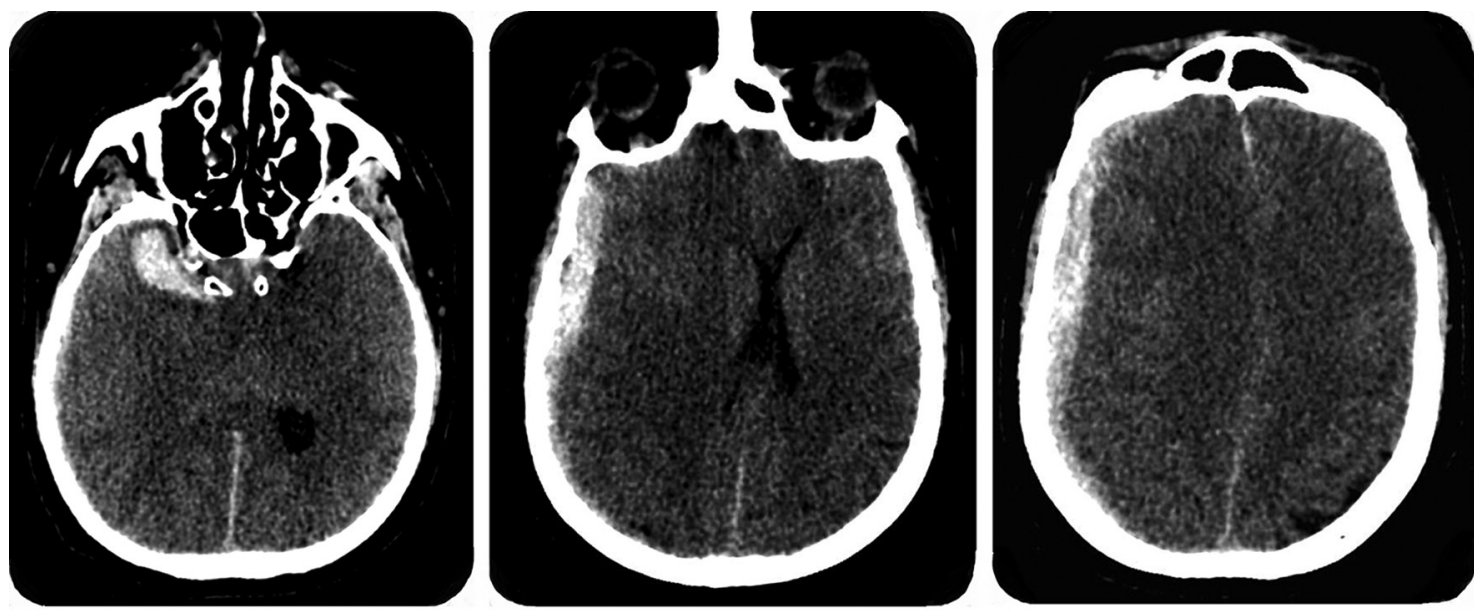

Fig. 1 The brain computed tomography shows a right acute subdural hematoma and a temporal hematoma.
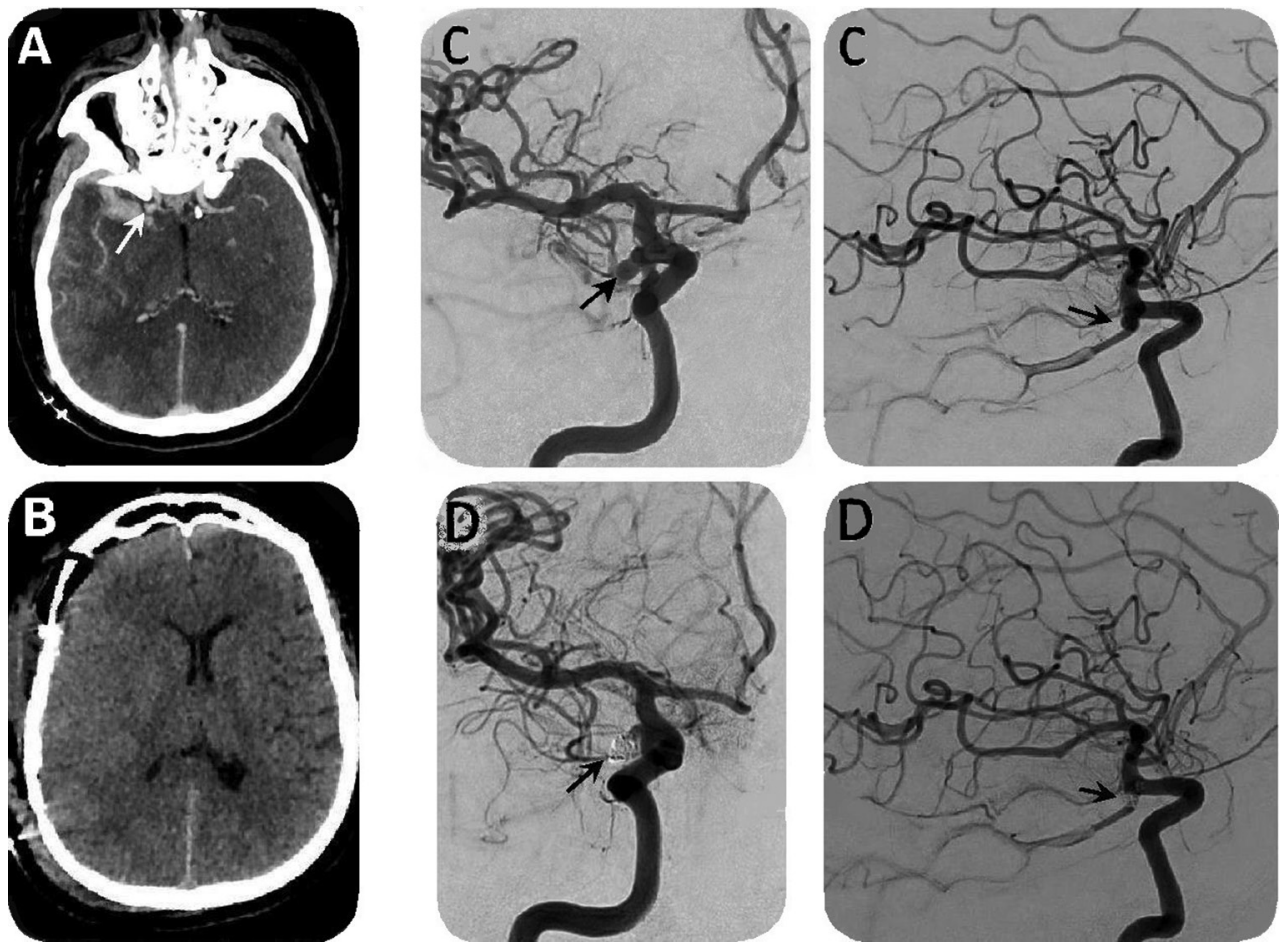

Fig. 2 (A) Preoperative computed tomography angiography shows the aneurysm of the right posterior communicating artery, (B) postoperative computed tomography, (C) digital subtraction angiography before aneurysm treatment, and (D) digital subtraction angiography after successful aneurysm endovascular coiling.

treated with coil embolization. The patient was subsequently admitted to the intensive care unit (ICU) for a ruptured aneurysm associated with ASH. Despite the presence of levetiracetam, he was unresponsive during initial resuscitation and was found to be in status epilepticus, which was managed with phenytoin and sedation. After 1 week in the ICU, the patient regained consciousness. His neurologic examination steadily improved to a GCS of 14 . The rest of his postoperative course was uneventful; he did not suffer from vasospasm, rebleeding, acute hydrocephalus, or electrolyte disturbances. After a few months of neurologic rehabilitation, he had residual mild disability classified as fourth-fifths on the Glasgow Outcome Scale, mostly due to relational disorders and aggressiveness (-Fig. 2).

\section{Discussion}

Atraumatic "spontaneous ASH" 1 is a rare complication of ruptured aneurysm ${ }^{2}(0.5-7.9 \%$ of cases), though aneurysm rupture is still the leading cause of spontaneous ASH, accounting for $76 \%$ of documented cases. ${ }^{3}$ Three different mechanisms have been proposed for aneurysm rupture resulting in spontaneous ASH. ${ }^{1,45}$ Prior bleeding leads to adhesions between the aneurysm sac and the arachnoid membrane, and the next aneurysm rupture egresses directly into the subdural space. ${ }^{2}$ Massive high-pressure bleeding breaks through the arachnoid membrane into the subdural space. ${ }^{3}$ A compressive ICH tears the covering cortex and arachnoid membrane. Considering the presence of an ICH of the right temporal pole, the 
third mechanism is a possible explanation in our case report. Nevertheless, PCOM aneurysm lies completely in the perimesencephalic cistern; thus, the first and the second mechanisms are possibly also involved, despite the absence of previous headaches and the lack of evidence of SAH on the CT.

The PCOM is the most frequent aneurysm location (30\%) that leads to ASH, followed by middle cerebral artery (MCA) aneurysms (27\%), anterior communicating artery (13\%), or anterior cerebral artery (11\%), rarely cortical aneurysms (4\%). The other etiologies of spontaneous ASH include bleeding from cortical branches of MCA (16\%) and finally tumors (8\%), such as metastasis, gliomas, and meningiomas. ${ }^{3}$ A patient suffering from spontaneous ASH generally presents to the emergency department for "acute-onset neurological impairment." Thus, it can be clinically mistaken for more common etiologies, such as head trauma or stroke. Oftentimes, the CT imaging obtained is interpreted by a general radiologist rather than one that specializes in neuroimaging. ${ }^{6}$

It is critical to obtain a thorough patient history with particular attention to the mechanism of injury (fall, trauma, alcohol intake, and anticoagulation status), clinical examination (wounds and cephalohematoma), and imaging (traumatic SAH, brain contusions, petechiae, and contrecoup injury). The absence of a clear mechanism of injury should raise suspicion for spontaneous ASH. Spontaneous ASH is often associated with ICH and/or SAH on head CT, though there are rare reported cases of "pure spontaneous ASH" where it can present in the absence of these signs. ${ }^{7,8}$

Initial management of spontaneous ASH is similar to the management of traumatic ASH, with particular emphasis on finding the cause of bleeding to avoid another hemorrhagic

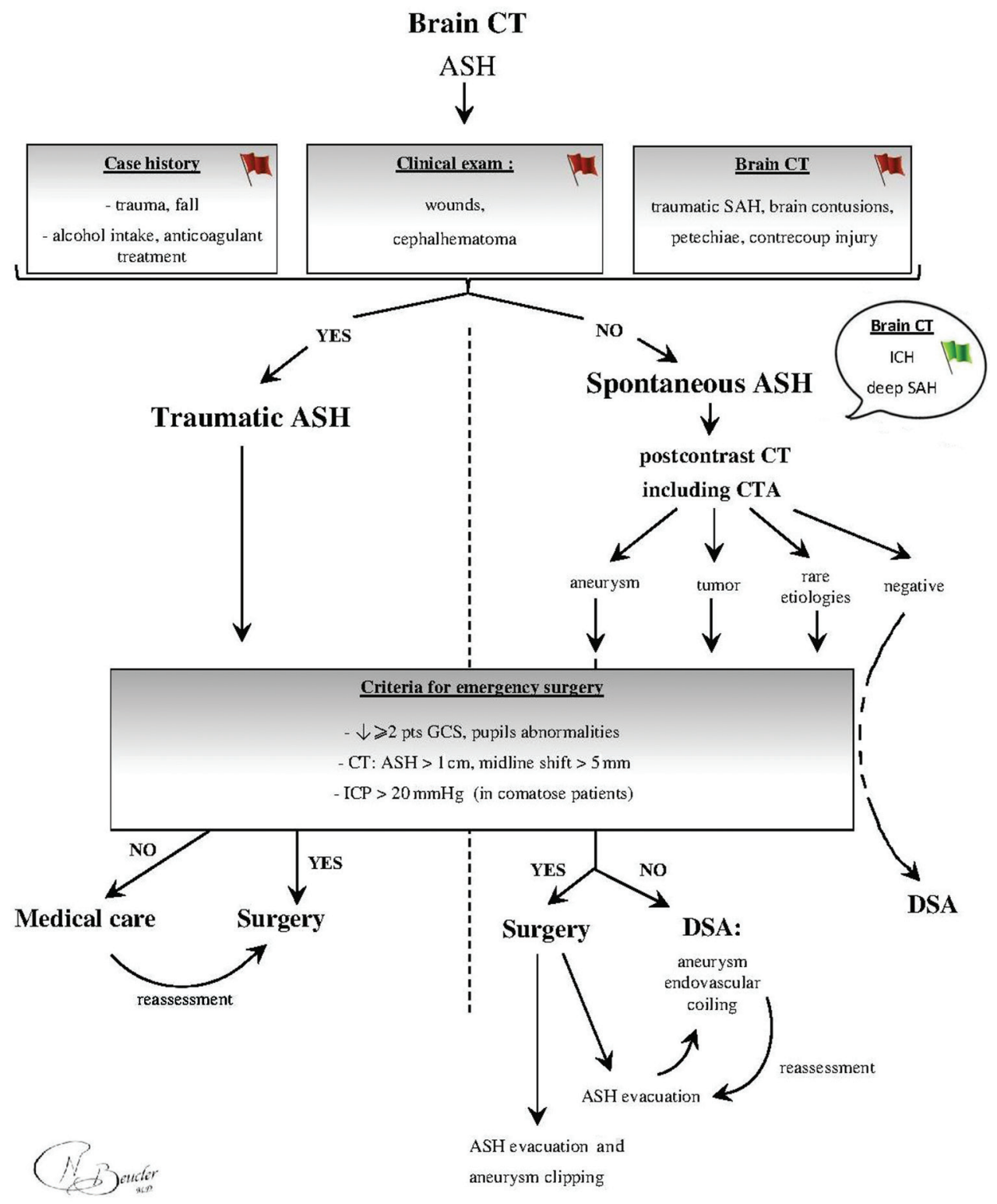

Fig. 3 Decision tree on the admission of a patient suffering from acute subdural hematoma. ASH, acute subdural hematoma; CTA, computed tomography angiography; DSA, digital subtraction angiography; ICH, intra cerebral hematoma; ICP, intracranial pressure; SAH, subarachnoid haemorrhage (Image courtesy: Dr. Nathan Beucler). 
event. Thus, a postcontrast CT, including CTA, is essential to identify an aneurysm, a tumor, or any other rare cause of spontaneous ASH. It is a sensitive, specific, rapid, and cost-effective examination, and it is widely accepted in the literature as a gold standard for workup of a ruptured aneurysm. ${ }^{9,10}$ Nevertheless, its timing in the management of patients suffering from ASH due to an aneurysm rupture is still debated in recent publications, ${ }^{11,12}$ especially when an emergency surgery is needed. We consider it as necessary for the future care of the patient, regardless of the severity of his clinical and radiological findings on admission. Thereafter, a neurosurgical opinion is necessary to assess the need for an emergency surgical intervention, ${ }^{13}$ depending on clinical and radiological severity criteria. ${ }^{14}$ These criteria include clinical findings, such as a drop of at least 2 points on the GCS, pupil abnormalities, intracranial pressure $>20 \mathrm{~mm} \mathrm{Hg}$ in comatose patients, and patients with radiologic severity evidence, such as ASH thickness $>10 \mathrm{~mm}$, or midline shift $>5 \mathrm{~mm}$. The presence of an aneurysm on the CTA enables the surgeon to perform a single-stage procedure for both ASH evacuation and aneurysm clipping. There is a slightly higher risk of complications from clipping, ${ }^{15,16}$ so this procedure should be performed by experienced surgeons, otherwise interventional radiology should be consulted for coiling. The surgery is optimally performed within the first 4 hours of symptoms onset. ${ }^{17,18}$ If the surgeon is able to evacuate the ASH and to clip the aneurysm during the same operative period, then the patient is transferred to ICU. Conversely, if the surgery is only devoted to the hematoma evacuation, the patient has to go through endovascular aneurysm coiling. If a patient with spontaneous ASH does not meet the clinical and the radiologic criteria for an emergency surgical procedure, the management of the aneurysm by endovascular coiling becomes the priority. Thereafter, any deterioration of a patient's clinical or radiologic status would lead to an immediate decompressive surgery ( - Fig. $\mathbf{3}$ ).

The other etiologies of spontaneous ASH, such as vessel bleeding and tumors, are not covered in this work. Concerning the rare etiologies of spontaneous ASH, postcontrast CT and CTA have a lack of sensitivity for very particular lesions, such as arteriovenous fistulas and small pial arteriovenous malformations. ${ }^{19}$ Therefore, spontaneous ASH with negative noninvasive imaging techniques should undergo a digital subtraction angiography which is the gold standard to diagnose these uncommon lesions.

Finally, spontaneous ASH from an aneurysm rupture is usually associated with SAH. Once the ASH has been managed and the aneurysm secured, the patient needs proper surveillance and treatment during at least 3 weeks as for any other kind of serious SAH, according to widely accepted guidelines. ${ }^{20}$

\section{Funding \\ None.}

\section{Conflict of Interest}

None declared.

\section{References}

1 Strauss I, Globus JH, Ginsburg SW. Spontaneous subarachnoid hemorrhageits to aneurysms of cerebral blood vessels. Arch Neur Psych 1932;27(5):1080-1132

2 Clarke E, Walton JN. Subdural haematoma complicating intracranial aneurysm and angioma. Brain 1953;76(3):378-404

3 Shekarchizadeh A, Masih S, Reza P, Seif B. Acute subdural hematoma and subarachnoid hemorrhage caused by ruptured cortical artery aneurysm: Case report and review of literature. Adv Biomed Res 2017;6:46

4 Weir B, Myles T, Kahn M, et al. Management of acute subdural hematomas from aneurysmal rupture. Can. J Neurol Sci 1984;11(3):371-376

5 Koerbel A, Ernemann U, Freudenstein D. Acute subdural haematoma without subarachnoid haemorrhage caused by rupture of an internal carotid artery bifurcation aneurysm: case report and review of literature. Br J Radiol 2005;78(931):646-650

6 Inamasu J, Saito R, Nakamura Y, et al. Acute subdural hematoma caused by ruptured cerebral aneurysms: diagnostic and therapeutic pitfalls. Resuscitation 2002;52(1):71-76

7 Junaid M, Bukhari SS, Kalsoom A, Afsheen A. Subdural hematomas following intracranial aneurysm rupture: A rare phenomenon. J Coll Physicians Surg Pak 2015;25(8):615-618

8 Thapa A, Kc B, Shakya B. Pure acute-on-chronic subdural hematoma due to ruptured posterior communicating artery aneurysm: Unsuspecting entity. World Neurosurg 2018; $114: 335-338$

9 Jayaraman MV, Mayo-Smith WW, Tung GA, et al. Detection of intracranial aneurysms: multi-detector row CT angiography compared with DSA. Radiology 2004;230(2):510-518

10 Yang ZL, Ni QQ, Schoepf UJ, et al. Small intracranial aneurysms: Diagnostic accuracy of CT angiography. Radiology 2017;285(3):941-952

11 Mrfka M, Pistracher K, Augustin M, Kurschel-Lackner S, Mokry M. Acute subdural hematoma without subarachnoid hemorrhage or intraparenchymal hematoma caused by rupture of a posterior communicating artery aneurysm: case report and review of the literature. J Emerg Med 2013;44(6):e369-e373

12 Mathais Q, Esnault P, Dagain A, et al. Spontaneous pure subacute subdural haematoma without subarachnoid haemorrhage caused by rupture of middle cerebral artery aneurysm. Anaesth Crit Care Pain Med 2018;37(6):623-624

13 O'Sullivan MG, Whyman M, Steers JW, Whittle IR, Miller JD. Acute subdural haematoma secondary to ruptured intracranial aneurysm: diagnosis and management. $\mathrm{Br} \mathrm{J}$ Neurosurg 1994;8(4):439-445

14 Bullock MR, Chesnut R, Ghajar J, et al. Surgical Management of Traumatic Brain Injury Author Group. Surgical management of acute subdural hematomas. Neurosurgery 2006;58(3, Suppl):S16-S24, discussion Si-iv

15 Molyneux A, Kerr R, Stratton I, et al; International Subarachnoid Aneurysm Trial (ISAT) Collaborative Group. International Subarachnoid Aneurysm Trial (ISAT) of neurosurgical clipping versus endovascular coiling in 2143 patients with ruptured intracranial aneurysms: a randomized trial. J Stroke Cerebrovasc Dis 2002;11(6):304-314

16 Hsu CE, Lin TK, Lee MH, et al. The impact of surgical experience on major intraoperative aneurysm rupture and their consequences on outcome: A multivariate analysis of 538 microsurgical clipping cases. PLoS One 2016;11(3):e0151805

17 Seelig JM, Becker DP, Miller JD, Greenberg RP, Ward JD, Choi SC. Traumatic acute subdural hematoma: major mortality reduction in comatose patients treated within four hours. N Engl J Med 1981;304(25):1511-1518 
18 Wilberger JE Jr., Harris M, Diamond DL. Acute subdural hematoma: morbidity, mortality, and operative timing. J Neurosurg 1991;74(2):212-218

19 Ding D, Bok AP. Acute subdural hematoma from a ruptured aneurysm of the distal middle cerebral artery. J Neurosci Rural Pract 2017;8(1):152-154

20 Connolly ES Jr., Rabinstein AA, Carhuapoma JR, et al; American Heart Association Stroke Council; Council on
Cardiovascular Radiology and Intervention; Council on Cardiovascular Nursing; Council on Cardiovascular Surgery and Anesthesia; Council on Clinical Cardiology. Guidelines for the management of aneurysmal subarachnoid hemorrhage: a guideline for healthcare professionals from the American Heart Association/american Stroke Association. Stroke 2012;43(6):1711-1737 